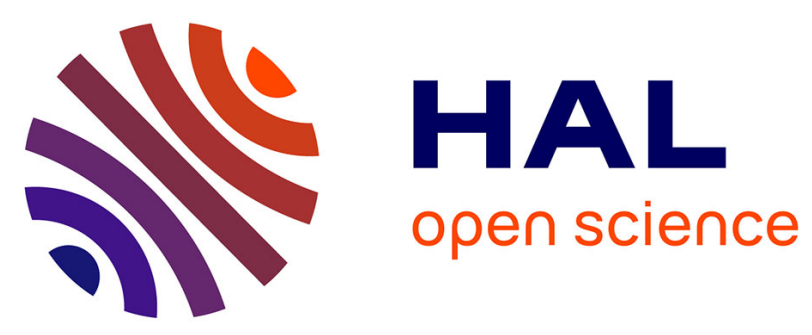

\title{
Radiomic analysis of HTR-DCE MR sequences improves diagnostic performance compared to BI-RADS analysis of breast MR lesions
}

\author{
Saskia Vande Perre, Loïc Duron, Audrey Milon, Asma Bekhouche, Daniel \\ Balvay, François Cornélis, Laure Fournier, Isabelle Thomassin-Naggara
}

\section{To cite this version:}

Saskia Vande Perre, Loïc Duron, Audrey Milon, Asma Bekhouche, Daniel Balvay, et al.. Radiomic analysis of HTR-DCE MR sequences improves diagnostic performance compared to BI-RADS analysis of breast MR lesions. European Radiology, 2021, 10.1007/s00330-020-07519-9 . hal-03160371

\section{HAL Id: hal-03160371 \\ https://hal.sorbonne-universite.fr/hal-03160371}

Submitted on 5 Mar 2021

HAL is a multi-disciplinary open access archive for the deposit and dissemination of scientific research documents, whether they are published or not. The documents may come from teaching and research institutions in France or abroad, or from public or private research centers.
L'archive ouverte pluridisciplinaire HAL, est destinée au dépôt et à la diffusion de documents scientifiques de niveau recherche, publiés ou non, émanant des établissements d'enseignement et de recherche français ou étrangers, des laboratoires publics ou privés. 


\section{RADIOMIC ANALYSIS OF HTR-DCE MR SEQUENCES IMPROVES DIAGNOSTIC PERFORMANCE COMPARED TO BI-RADS SCORE ANALYSIS OF BREAST MR LESIONS}

\section{ABSTRACT}

PURPOSE: To assess the diagnostic performance of radiomic analysis using High Temporal Resolution (HTR)-Dynamic Contrast Enhancement (DCE) MR sequences compared to BIRADS analysis to distinguish benign from malignant breast lesions.

MATERIALS AND METHODS: We retrospectively analyzed data from consecutive women who underwent breast MRI including HTR-DCE MR sequencing for abnormal enhancing lesions and who had subsequent pathological analysis at our tertiary center. Semiquantitative enhancement parameters and textural features were extracted. Temporal change across each phase of textural features in HTR-DCE MR sequences were calculated and called "kinetic textural parameters". Statistical analysis by LASSO-logistic regression and cross validation were performed to build a model. The diagnostic performance of the radiomic model was compared to the results of BI-RADS MR score analysis.

RESULTS: We included 117 women with a mean age of 54 years (28-88). Of the 174 lesions analyzed 75 were benign and 99 malignant. Seven semi-quantitative enhancement parameters and 57 textural features were extracted. Regression analysis selected 15 significant variables in a radiomic model (called "malignant probability score") which displayed an AUC=0.876 (sensitivity $=0.98$, specificity $=0.52$, accuracy $=0.78$ ). The performance of the malignant probability score to distinguish benign from malignant breast lesions (AUC $=0.876,95 \% \mathrm{CI}$ 0.825-0.925) was significantly better than that of BI-RADS analysis (AUC $=0.831,95 \% \mathrm{CI}$ 0.769-0.892). The radiomic model significantly reduced false positives (42\%) with the same number of missed cancers $(\mathrm{n}=2)$ 
CONCLUSION: A radiomic model including kinetic textural features extracted from an HTR-DCE MR sequence improves diagnostic performance over BI-RADS analysis.

\section{KEY WORDS:}

Breast

Neoplasms

MRI Image enhancement

Artificial intelligence

\section{KEY POINTS:}

1- Radiomic analysis using HTR-DCE is of better diagnostic performance [AUC $=0.876$ ] than conventional breast MRI reading with BI-RADS [AUC $=0.831](\mathrm{p}<0.001)$

2- A radiomic malignant probability score under $19.5 \%$ gives a negative predictive value of $100 \%$ while a malignant probability score over $81 \%$ gives a positive predictive value of $100 \%$

3- Kinetic textural features extracted from HTR-DCE MRI have a major role to play in distinguishing benign from malignant breast lesions. 


\section{ABBREVIATION AND ACRONYMS}

AUC: Area Under the receiver operating characteristic Curve

BI-RADS: Breast Imaging Reporting And Data System

CCC: Concordance Correlation Coefficient

CEROG : Comité d'Ethique de la Recherche en Obstetrique et Gynecologie

DCE: Dynamic Contrast Enhancement

DCIS: Ductal Carcinomas in situ

DISCO: Differential Subsampling with Cartesian Ordering

EA: Enhancement Amplitude

EI: Enhancement Integral

GLCM: Grey-Level Co-occurrence Matrix

GLDM: Grey-Level Dependence Matrix

GLRLM: GLevel Run-Length Matrix

GLSZM: Grey-Level Size-Zone Matrix

HTR: High Temporal Resolution

ICC: Intra-class Correlation Coefficient

IDC: Invasive Ductal Carcinoma

Imc1: Informational measure of correlation 1

LASSO: Least Absolute Shrinkage and Selection Operator

MR: Magnetic Resonance

MSI: Maximum Slope of Increase

PACS: Picture Archiving and Communication System

RLNN: Run Length Non-uniformity Normalized

Rmax: Maximum of enhancement

RmaxTiming: Timing of Maximum of enhancement

ROC: Receiver Operating Characteristic

ROI: Region Of Interest

SPGR: Spoiled Gradient Recalled

STD: Standard Deviation of signal intensity

THR: Time of Half Rising

WIR: Wash-In-Rate 


\section{INTRODUCTION}

For many years, breast magnetic resonance (MR) imaging was acquired at low temporal resolution to allow high spatial resolution reaching $1 \mathrm{~mm}$ voxel size $(1,2)$. Although this conventional protocol has a high sensitivity (89-100\%), it is hampered by a variable specificity (35\%-64\%) mainly depending on the indication (3). With the development of new k-space sampling techniques, high temporal resolution (HTR)-dynamic contrast enhancement (DCE) MR sequencing has become feasible in breast MR imaging opening up the possibility of using early DCE characteristics of breast tumors. In this setting, Mann et al.(4) described a correlation between maximum slope of enhancement during the first minute after injection with tumor grade and suggested that this criteria optimized differentiation between benign and malignant lesions. More recently, Milon et al. demonstrated the value of heterogeneity early after gadolinium injection based on the analysis of HTR-DCE MR sequences. Malignant lesions were more heterogeneous than benign lesions with a higher standard deviation of signal intensity (STD) irrespective of the tested rank of the HTR-DCE sequence (5).

Some authors have underlined that in various organs radiomic analysis of spatial heterogeneity (i.e. textural features) improves tissue characterization (6-8). Others have taken this further and analyzed the change in textural parameters over acquisition time (9). Several studies have established the usefulness of radiomic analysis in breast MR imaging for diagnosis, prognosis and prediction of the therapeutic response (10-23). Most radiomic signatures were built on conventional breast MR imaging protocols (24-26). We developed a radiomic model combining spatial heterogeneity analysis using textural features and their changes during the first minute after gadolinium chelate injection on an HTR-DCE MR sequence. The study we present here retrospectively assessed the diagnostic performance of radiomic analysis using HTR-DCE MR sequences compared to BI-RADS analysis to distinguish benign from malignant breast lesions. 


\section{MATERIAL AND METHODS}

The study was approved by CEROG, an institutional ethics committee, and was not subject to informed consent (CEROG 2018-GYN-0803).

\section{Patient Population.}

The MR imaging database of Tenon Hospital (APHP, Paris, France) was retrospectively queried to identify all women who underwent breast MR imaging between $18^{\text {th }}$ July 2016 and $31^{\text {st }}$ March $2017(\mathrm{n}=520)$. Four hundred and three patients were excluded for the following reasons: no biopsy performed (negative or benign examination) $(n=364)$, breast MR biopsy procedure $(n=20)$; incomplete acquisition protocol $(n=11)$, biopsy-proven lesions without enhancement on MRI ( $n=3)$, problem related to the Picture Archiving and Communication System (PACS) with duplicate examination $(n=4)$, lesions of uncertain nature in a woman who underwent MRI for breast cancer follow-up with neoadjuvant chemotherapy $(n=1)$ (Figure 1).

The final population consisted of 117 women (mean age: 54 years, range 28-88) with 174 lesions. Menopausal women represented $61.5 \%$ of the overall population (72/117). Breast MR indications were breast cancer staging [73/117 (62.4\%)], problem-solving after inconclusive mammography and ultrasound assessment [23/117 (19.6\%)], high-risk screening [(9/117 (7.7\%)], surveillance of probably benign lesions (BI-RADS 3) [4/117 (3.4\%)], nipple discharge assessment [3/117 (2.6\%)], screening for primary cancer in women with a metastatic axillary lymphadenopathy [2/117 (1.7\%)], for neoadjuvant chemotherapy work-up [2/117 (1.7\%)], and for prophylactic mastectomy work-up [1/117 (0.9\%)].

\section{MRI acquisition.}

MRI sequences were acquired with patients in the prone position on a $1.5 \mathrm{~T}$ Optima MR450w GEM system using a phased array dedicated breast coil 8-channel (GE, Chicago, 
Ilinois, USA). The protocol is detailed in Supplementary Material 1. Eleven acquisitions with a temporal resolution of $7.7 \mathrm{~s}$ per acquisition that corresponded to the HTR-DCE sequence were added to this standard protocol during the first $1 \mathrm{~min} 18 \mathrm{~s}$ following Gadolinium Chelate injection (Dotarem $0.5 \mathrm{mmol} / \mathrm{mL}$; Guerbet, Villepinte, France ; $0.2 \mathrm{~mL} . \mathrm{kg}-1$ body weight) via a power injector at a rate of $2 \mathrm{~mL} . \mathrm{s}-1$ followed by $20 \mathrm{~mL}$ saline flush . This sequence, called Differential Subsampling with Cartesian Ordering (DISCO), is a T1 dualecho 3D spoiled gradient recalled (SPGR) acquisition sequence with a pseudorandom variable density k-space segmentation using an elliptical model where the central k-space regions are acquired more frequently than the peripheral region and a view-sharing reconstruction (27).

\section{BI-RADS analysis}

Two radiologists $(\mathrm{AB}, \mathrm{AM})$, blinded to the clinical data, indication and pathological results, with 6 and 2 years of experience respectively in breast MR imaging, independently reviewed the images according to the BI-RADS lexicon (28) on the standard protocol (Supplementary Material 2). A receiver operator characteristic (ROC) curve analysis was performed to compare the BI-RADS final score versus the radiomic model using De Long et al's method (29). For the diagnostic performance analysis, BI-RADS 3 lesions were considered as probably benign (diagnostic study) and BI-RADS 4 and 5 considered as suspicious or probably highly suspicious (Supplementary Material 2). In case of disagreement, the score allocated by the most experienced reader was retained.

\section{Extraction of radiomic features}

\section{1) Semi-quantitative enhancement features.}

Enhancement curves were extracted from AW server software (GE, Chicago, Ilinois USA) after a two-dimensional manual delineation. The following parameters were extracted 
directly from the curves (Figure 2): Enhancement Integral (EI (\%), Maximum Slope of Increase [MSI (\%/sec)], Maximum of enhancement [Rmax (\%)], Timing of Maximum of enhancement [RmaxTiming $(\mathrm{sec})]$ and Wash-In-Rate [(WIR $(\% / \mathrm{sec})]$. The curves were then fitted using a Hill equation $\mathrm{EI}(\mathrm{t})=\frac{\mathrm{A}}{\left(1+\left(\frac{\mathrm{B}}{\mathrm{t}}\right) \mathrm{C}\right)}$ (also known as sigmoid function) allowing other semi-quantitative parameters as previously described (30), where $\mathrm{A}$ is the asymptotic enhancement amplitude (EA), B is the time of half rising (THR), and C is a power constant (31) using Matlab Software version R2016a (The MathWorks Inc., Natick, Massachusetts, USA).

\section{2) Non-kinetic textural features}

Segmentation: Two radiologists (SVP, LD), blinded to medical history and histological results, performed a two-dimensional manual delineation on the HTR-DCE sequence using the ITK-SNAP software, University of Pennsylvania, USA (version 3.6) (32).The regions of interest (ROI) were then propagated to all the timepoints of the sequence.

Feature extraction: Textural features were extracted using the Pyradiomics open source software, Harvard medical school, Boston, Massachusetts, USA (33) according to the definitions of the Image Biomarker Standardization Initiative (34) and an absolute discretization with fixed bin size model with 25 grey levels (35). For each of the 11 phases of the HTR-DCE MR sequence, the ROI provided 104 radiomic features including 16 shapebased features, 19 first-order statistics and 69 textural features derived respectively from the grey-level co-occurrence matrix obtained using 4 angles (GLCM, 23 features), grey-level runlength matrix (GLRLM, 16 features), grey-level size-zone matrix (GLSZM, 16 features) and grey-level dependence matrix (GLDM, 14 features).

Feature reduction according to observer reproducibility: A first step of feature reduction was carried out to select parameters which were less sensitive to variation of manual segmentation. All the lesions were segmented 3 times: once by a radiologist (LD) and then 
twice by another radiologist (SVP) 2 weeks later. By means of the R-3.3.3 (R Foundation, Vienna, Austria) (36), we calculated the intra-class correlation coefficient (ICC) on all pair combinations of readings, and a concordance correlation coefficient (CCC) on the intraobserver pair, according to a previously published methodology (35). A feature was selected if all three ICC values were $\geq 0.8$ and the CCC value $\geq 0.9$ (Figure 3). Fifty-seven texture features were considered as reproducible (six morphological features, 15 first-order texture features, 36 second-order features) (Table 1) for each phase. Thus, 627 non-kinetic textural features were selected from the first segmentation of the radiologist who segmented the lesions twice (SVP).

$\underline{\text { Feature analysis }}$ A Least Absolute Shrinkage and Selection Operator (LASSO) regression (37) with a penalization coefficient $\lambda$ ranging from 0.02 to 0.04 was performed separately for each phase of the HTR-DCE MR sequence with the 57 textural features previously selected. The area under the curve (AUC) of the LASSO resulting from each phase was calculated and compared.

\section{3) Textural kinetic analysis}

\section{Feature extraction}

We calculated the mean, minimum, maximum, median, and variance values of the 57 different textural features over time acquisition. Changes were assessed by the growth rate defined by $\frac{\text { Phase }_{x+1}-\text { Phase }_{x}}{\text { Phase }_{x}}$ and volatility defined by Phase $e_{x+1}-$ Phase $_{x}$ and their mean, maximum and minimum values. Thus, we obtained 11 textural kinetic features for each of the 57 textural features, i.e., 627 kinetic textural features.

\section{Feature reduction}


A five-run LASSO regression with a penalization coefficient $\lambda$ ranging from 0.045 to 0.058 was performed to preselect the more stable kinetic textural features. The rule "selected at least twice by these five runs of LASSO" was used to select the kinetic textural features.

\section{Reference standard}

\section{Histological}

Biopsies were performed for suspicious lesions (BI-RADS 4-5) or for some BI-RADS 3 lesions depending on the clinical context. For lesions with a sonographic correlation, biopsies were performed under sonography with an automatic 14-gauge core needle [74\% of lesions (128/174)] or with a vacuum-assisted core needle 7- to 10-gauge [2\% (4/174)]. Three lesions $[1.7 \%(3 / 174)]$ had a fine needle aspiration. For lesions without sonographic correlation, biopsies were performed with a vacuum-assisted core needle 7 - to 10-gauge either under stereotactic guidance in $11 \%(19 / 174)$ or under MR guidance in $10 \%(18 / 174)$. One lesion was investigated directly by surgical biopsy guided radiologically, and information on how the biopsy was performed was unavailable for one. The maximum interval between breast MR imaging and biopsy was 4 months. Reference standard corresponded to histopathological findings of percutaneous biopsies for benign lesions $(n=68)$ or of surgery for high-risk lesions $(\mathrm{n}=7)$ or malignant tumors $(\mathrm{n}=99)$ including 16 pure ductal carcinomas in situ (DCIS). Final excision histology results are summarized in Supplementary Material 3. 


\section{RESULTS}

\section{BI-RADS and semi-quantitative enhancement curve analysis}

Breast density was evaluated as type A in $8.5 \%$ (10/117), type B in $44.4 \%(52 / 117)$, type C in $34.2 \%(40 / 117)$ and type D in $10.3 \%(12 / 117)$. Three patients $(2.6 \%, 3 / 17)$ had a bilateral mastectomy. The lesions had a mean size of $24.8 \mathrm{~mm}$ ( 3 to $100 \mathrm{~mm}$ ) and were a mass in $68.4 \%(119 / 174)$, a non-mass enhancement in $27 \%(47 / 174)$, a focus in $2.9 \%(5 / 174)$ or an atypical cyst in $1.7 \%(3 / 174)$.

The sensitivity, specificity, positive likelihood ratio, negative likelihood ratio and accuracy are detailed in Table 2 . The positive predictive values of BI-RADS 3, 4A, 4B, 4C and 5 were $13.33 \%(2 / 15), 24.24 \%(8 / 33), 37.5 \%(12 / 32), 69.04 \%(29 / 42)$ and $92.31 \%$ (48/52), respectively, with an AUC of 0.831 (95\%CI : 0.769-0.892, p<0.001).

\section{Radiomic analysis}

\section{$\underline{\text { Textural features: }}$}

Eleven LASSO were performed (one for each phase of HTR-DCE MR sequence). Second-order textural features (included in GLCM, GLRLM and GLDM) were used by all 11 LASSO (Supplementary Material 4). The feature "maximum diameter" was selected in 7/11 LASSO. The first-order textural features " $10^{\text {th }}$ percentile of signal intensity" and "uniformity" were selected by 2/11 LASSO. ROC AUC for predicting malignancy ranged from 0.76 to 0.80. according to the phase (Figure 4). Each phase model displayed a lower AUC value (0.76 to 0.80$)$ than that of BI-RADS analysis (AUC $=0.83$ )

\section{$\underline{\text { Kinetic textural features: }}$}

Ten of the 627 kinetic textural features were selected by the rule "selected at least twice by the five LASSO runs" and were included in the final model (Supplementary Material 5). 


\section{Final model - Building and validation}

Finally, we used a LASSO regression with cross-validation with results expressed as a “probability score of malignancy" from $0 \%$ to $100 \%$. LASSO was applied on the pre-selected kinetic textural features $(n=10)$ and on the semi-quantitative enhancement parameters $(n=7)$ with a penalization coefficient $\lambda$ of 0.005 (Figure 3).

Fifteen parameters were retained in the final model including first-order texture kinetics $(n=2)$, second-order texture kinetics $(n=8)$ and semiquantitative parameters $(n=5)$. The selected variables and the LASSO coefficient are listed in Table 3. The three variables with the highest LASSO coefficient were all second-order textural features: the median value of the informational measure of correlation 1 (Imc1) extracted from the GLCM (coefficient 6.789), the Minimum growth rate of the Dependence Non-Uniformity from the GLDM (coefficient 35.16) and the Minimum volatility of the Run Length Non-Uniformity Normalized (RLNN) from the GLRLM (coefficient 19.76). The AUCs of each of these variables for the diagnosis of malignancy were respectively $0.630(\mathrm{p}=0.003,95 \% \mathrm{CI}: 0.546$ 0.714), 0.714 ( $\mathrm{p}<0.0001,95 \%$ CI: 0.637-0.792), and 0.681 ( $\mathrm{p}<0.0001,95 \%$ CI: $0.597-0.765)$.

This final model displayed an AUC=0.876 (95\%CI: 0.825-0.925). With a threshold fixed at $28.4 \%$ (i.e. lesions with a malignant probability score less than $28.4 \%$ are classified as benign), the model yielded a sensitivity of $98 \%$ (97/99), specificity of 52\% (39/75), and accuracy of 78\% (136/174) (Table 2). A model with a threshold probability of malignancy calculated under $19.5 \%$, has a negative predictive value of $100 \%(\mathrm{n}=31)$. Similarly, a model with a threshold probability of malignancy calculated above $81 \%$, predicted malignancy in $100 \%$ [i.e. positive predictive value of $100 \%(n=48)]$.

Changes in the probability score of malignancy as a function of the diameter of the lesion is represented in Figure 5 showing that no correlation exists between these two variables. 
Finally, we tested the quality of the model applying the Radiomics Quality Score (RQS) (38) and obtained an intermediate score level of 18/36 (Supplementary Material 6).

\section{Comparison between radiomic model and BI-RADS analysis}

The performance of the radiomic model to distinguish between benign and malignant breast lesions (AUC $=0.876,95 \% \mathrm{CI}$ : $0.825-0.925$ ) was significantly better than that of the BIRADS analysis (AUC $=0.831,95 \% \mathrm{CI}$ : 0.769-0.892) (Figure 6). Both the radiomic model and BI-RADS analysis displayed the same high sensitivity (0.98) with only two false negatives. However, two infiltrative cancers (one invasive lobular carcinoma and one mucinous carcinoma) were missed with the BI-RADS analysis while only one DCIS and one invasive ductal carcinoma (IDC) were missed by the malignancy probability score. Finally, the specificity of the radiomic model was much higher than that of BI-RADS analysis (52\% versus 17\%) with a lower number of false positives (36 versus 62) (Figure 6). 


\section{DISCUSSION}

Our study demonstrates that radiomic analysis based on HTR-DCE has a better diagnostic performance $[\mathrm{AUC}=0.876$ (IC 0.825-0.925)] than conventional breast MR imaging reading with BI-RADS [AUC $=0.831 \quad(95 \% \mathrm{CI}$ : 0.769-0.892, $\mathrm{p}<0.001)$ ]. Moreover, the radiomic model, called the "malignant probability score", reduces the number of false positives of breast MR imaging by $42 \%$ (36 versus 62 ). Finally, a malignant probability score under $19.5 \%$ gives a negative predictive value of $100 \%$ (no cancer was missed) and a malignant probability score over $81 \%$ gives a positive predictive value of $100 \%$ (no false positive).

Our study demonstrates the diagnostic value of textural analysis of heterogeneity for improving tissue characterization. The application of radiomics in breast cancers is an emerging translational research topic as witnessed by an exponential growth in the number of studies on diagnosis (detection or characterization) or prognosis (prediction of morbidity, mortality or response to therapy). Most radiomic approaches in breast imaging to date have focused on interrogating heterogeneity patterns across the entire tumor. Parekh et al. (6) demonstrated that breast cancers have a higher entropy on DCE MR than benign lesions or glandular tissue. Other authors have investigated the usefulness of radiomics in recognizing different molecular types of cancer based on the heterogeneity of textural features such as skewness and kurtosis (24), or the expression of Ki67 for which Liang et al. submitted a radiomics classification using a LASSO regression with an AUC of 0.740 (39). In our study, textural kinetic features clearly improved the diagnostic performance as the phase-by-phase radiomic model did not show any superiority compared to the BI-RADS score. The parameters most used by the final model came from complex textural matrices indistinguishable to the naked eye, i.e., kinetic textural features resulting from second-order matrices: the median value of Imc1 extracted from the GLCM, the minimum growth rate of 
the dependence non-uniformity from the GLDM, and the minimum volatility of the RLNN from the GLRLM.

The originality of our results is that we demonstrate that radiomic analysis applied to HTR-DCE MR sequence helps improve breast MR specificity by decreasing false positive results by $42 \%$. HTR-DCE MR sequences are particularly useful in abbreviated protocols which are becoming more widespread along with the increase in the number of indications for breast MR imaging as a screening tool $(40,41)$. Several authors have demonstrated that the first part of the dynamic curve (especially the maximum slope) may help improve characterization in a protocol without conventional time-intensity curve analysis (42). Classically, maximum slope cannot be extracted from a standard breast MRI protocol where the first sequence is acquired more than one minute after gadolinium injection (43). DCE MR sequences with high temporal resolution can assess the perfusion abnormalities inherent to neo-angiogenesis often induced by cancer cells (44). The tumor vascular network displays permeability anomalies (well assessed with a conventional breast protocol), perfusion abnormalities (loss of the arteriole-capillary-venule pattern) and morphological abnormalities (architectural disorganization, irregular diameter) (45-47). Perfusion abnormalities may be assessed with HTR-DCE MR sequences while spatial heterogeneity related to morphological abnormalities requires analysis of textural features. The current study, was the first to combine these two characteristics by measuring texture variability in the different phases of the HTR-DCE MR sequences with a radiomic analysis (kinetic textural parameters) (48).

In the literature, analysis of heterogeneity over time in breast cancers remains underexplored. In 2011, Agner et al. described the value of kinetic textural parameters extracted from 41 breast DCE MR which, combined in a model with morphologic descriptors 
(i.e. smoothness), yielded an AUC of 0.91 with $99 \%$ sensitivity, $76 \%$ specificity, and $89 \%$ accuracy (49). Thibault et al. demonstrated the effectiveness of texture analysis on parametric maps calculated from DCE-MRI for early prediction of breast cancer response to neoadjuvant chemotherapy (50). In a study published by Fan et al (24) using radiomics on tumors and the surrounding parenchyma decomposed into subregions by their kinetics of enhancement on a conventional DCE-MRI protocol, the tumor subregion related to fast-flow kinetics had the strongest predictive power of cancer subtype with an AUC of 0.832 . However, these studies could only explore the wash-out textural kinetics due to the low temporal resolution of the conventional DCE-MRI protocol. Very few studies have applied radiomic analysis on an HTR DCE sequence and therefore studied the wash-in textural kinetics of breast cancer. Two studies used features extracted from a parametric map built on an HTR-DCE MR sequence: Milenković et al (51) built a model able to diagnose malignant lesions with a ROC AUC of 0.887 and Zhou et al (52) found AUC for the diagnosis of breast lesion for radiomic parameters calculated from Ktrans, Kep, Ve and Vp of 0.95, 0.93, 0.89 and 0.96, respectively. However, unlike our study, no comparison with human BI-RADS analysis was performed in either of these two studies. The model created in our study used semi-quantitative enhancement parameters and kinetic textural features extracted from the first 78 seconds after contrast injection. The coefficient given by the LASSO to these features resulting from second-order matrices highlights the interest of studying textural kinetics on HTR DCE sequences. Separately from the model, three kinetic textural features provide AUCs for malignancy diagnosis of 0.714 (minimum growth rate of the dependence non-uniformity from the GLDM), 0.681 (minimum volatility of the RLNN from the GLRLM), and 0.630 (median value of Imc1 from the GLCM).

This study validates a robust method with low variability using automatically extracted quantitative parameters. Quality assurance was better than in previous studies (53). 
Moreover, we applied a LASSO regression with cross validation to validate the model internally pending external validation.

Nevertheless, our study has several limitations. First, it was a retrospective preliminary clinical study and we did not perform a preliminary phantom study. Second, the very high disease prevalence in our study reflects the population consulting in our tertiary center and constitutes a selection bias. Our results remain to be confirmed in future research studies, especially in a screening population where abbreviated protocols are becoming standard (41). Furthermore, all the data were obtained from one center with a single MRI machine, software and protocol, and our results need to be externally validated probably using a harmonization of radiomic features method such as the ComBat method (54). Fourth, we only performed a cross validation of our results due to the relatively small sample size (174 lesions). External validation is thus necessary to validate these results in a different population with different technical parameters. Finally, we did not compare or combine HTR DCE MR with other abbreviated protocols based on DW Imaging. This combination of HTR DCE and DW could be interesting and will certainly be explored in further studies (55).

In conclusion, our study demonstrates the better diagnostic performance of radiomic analysis using kinetic textural and enhancement features extracted from HTR-DCE MR sequences to distinguish breast lesions compared to BI-RADS analysis. Further studies are now needed to evaluate the additional value of the combination of radiomic and human analysis.

\section{ACKNOWLEDGMENTS:}

Nicolas Mion Eng. and Julie Poujol, PhD 


\section{REFERENCES}

1. Sardanelli F, Boetes C, Borisch B, et al. Magnetic resonance imaging of the breast: recommendations from the EUSOMA working group. Eur J Cancer Oxf Engl 1990. 2010 May;46(8):1296-316.

2. Mann RM, Cho N, Moy L. Breast MRI: State of the Art. Radiology. 2019;292(3):52036.

3. Kuhl C. The current status of breast MR imaging. Part I. Choice of technique, image interpretation, diagnostic accuracy, and transfer to clinical practice. Radiology. 2007 Aug;244(2):356-78.

4. Mann RM, Mus RD, van Zelst J, Geppert C, Karssemeijer N, Platel B. A novel approach to contrast-enhanced breast magnetic resonance imaging for screening: highresolution ultrafast dynamic imaging. Invest Radiol. 2014 Sep;49(9):579-85.

5. Milon A, Vande Perre S, Poujol J, et al. Abbreviated breast MRI combining FAST protocol and high temporal resolution (HTR) dynamic contrast enhanced (DCE) sequence. Eur J Radiol. 2019 Aug 1;117:199-208.

6. Parekh VS, Jacobs MA. Integrated radiomic framework for breast cancer and tumor biology using advanced machine learning and multiparametric MRI. NPJ Breast Cancer. 2017;3:43.

7. Rizzo S, Botta F, Raimondi S, et al. Radiomics of high-grade serous ovarian cancer: association between quantitative $\mathrm{CT}$ features, residual tumour and disease progression within 12 months. Eur Radiol. 2018 Nov;28(11):4849-59.

8. Wu G, Woodruff HC, Sanduleanu S, et al. Preoperative CT-based radiomics combined with intraoperative frozen section is predictive of invasive adenocarcinoma in pulmonary nodules: a multicenter study. Eur Radiol. 2020 May;30(5):2680-91.

9. Thomassin-Naggara I, Soualhi N, Balvay D, Darai E, Cuenod C-A. Quantifying tumor vascular heterogeneity with DCE-MRI in complex adnexal masses: A preliminary study. J Magn Reson Imaging JMRI. 2017 Apr 3; 10.1002/jmri.25707

10. Chang Y-C, Huang C-S, Liu Y-J, Chen J-H, Lu Y-S, Tseng W-YI. Angiogenic response of locally advanced breast cancer to neoadjuvant chemotherapy evaluated with parametric histogram from dynamic contrast-enhanced MRI. Phys Med Biol. 2004 Aug 21;49(16):3593-602.

11. Ahmed A, Gibbs P, Pickles M, Turnbull L. Texture analysis in assessment and prediction of chemotherapy response in breast cancer. J Magn Reson Imaging JMRI. 2013 Jul;38(1):89-101.

12. Ashraf A, Gaonkar B, Mies C, et al. Breast DCE-MRI Kinetic Heterogeneity Tumor Markers: Preliminary Associations With Neoadjuvant Chemotherapy Response. Transl Oncol. 2015 Jun;8(3):154-62.

13. Bhooshan N, Giger ML, Jansen SA, Li H, Lan L, Newstead GM. Cancerous breast lesions on dynamic contrast-enhanced MR images: computerized characterization for imagebased prognostic markers. Radiology. 2010 Mar;254(3):680-90.

14. Kim J-H, Ko ES, Lim Y, et al. Breast Cancer Heterogeneity: MR Imaging Texture Analysis and Survival Outcomes. Radiology. 2017;282(3):665-75.

15. Parikh J, Selmi M, Charles-Edwards G, et al. Changes in primary breast cancer heterogeneity may augment midtreatment MR imaging assessment of response to neoadjuvant chemotherapy. Radiology. 2014 Jul;272(1):100-12.

16. Thibault G, Tudorica A, Afzal A, et al. DCE-MRI Texture Features for Early Prediction of Breast Cancer Therapy Response. Tomogr J Imaging Res. 2017 Mar;3(1):2332.

17. Wu J, Cao G, Sun X, et al. Intratumoral Spatial Heterogeneity at Perfusion MR 
Imaging Predicts Recurrence-free Survival in Locally Advanced Breast Cancer Treated with Neoadjuvant Chemotherapy. Radiology. 2018;288(1):26-35.

18. Wu J, Gong G, Cui Y, Li R. Intratumor partitioning and texture analysis of dynamic contrast-enhanced (DCE)-MRI identifies relevant tumor subregions to predict pathological response of breast cancer to neoadjuvant chemotherapy. J Magn Reson Imaging JMRI. 2016 Nov;44(5):1107-15.

19. Braman NM, Etesami M, Prasanna P, et al. Intratumoral and peritumoral radiomics for the pretreatment prediction of pathological complete response to neoadjuvant chemotherapy based on breast DCE-MRI. Breast Cancer Res BCR. 2017 May 18;19(1):57.

20. Fan M, Li H, Wang S, Zheng B, Zhang J, Li L. Radiomic analysis reveals DCE-MRI features for prediction of molecular subtypes of breast cancer. PloS One. 2017;12(2):e0171683.

21. Fan M, Cheng H, Zhang P, et al. DCE-MRI texture analysis with tumor subregion partitioning for predicting Ki-67 status of estrogen receptor-positive breast cancers. J Magn Reson Imaging JMRI. 2018 Jul;48(1):237-47.

22. Li H, Zhu Y, Burnside ES, et al. MR Imaging Radiomics Signatures for Predicting the Risk of Breast Cancer Recurrence as Given by Research Versions of MammaPrint, Oncotype DX, and PAM50 Gene Assays. Radiology. 2016 Nov;281(2):382-91.

23. Truhn D, Schrading S, Haarburger C, Schneider H, Merhof D, Kuhl C. Radiomic versus Convolutional Neural Networks Analysis for Classification of Contrast-enhancing Lesions at Multiparametric Breast MRI. Radiology. 2019 Feb;290(2):290-7.

24. Fan M, Zhang P, Wang Y, et al. Radiomic analysis of imaging heterogeneity in tumours and the surrounding parenchyma based on unsupervised decomposition of DCE-MRI for predicting molecular subtypes of breast cancer. Eur Radiol. 2019 Aug;29(8):4456-67.

25. Lo Gullo R, Daimiel I, Rossi Saccarelli C, et al. Improved characterization of subcentimeter enhancing breast masses on MRI with radiomics and machine learning in BRCA mutation carriers. Eur Radiol. 2020 Jun 27; 10.1007/s00330-020-06991-7

26. D’Amico NC, Grossi E, Valbusa G, et al. A machine learning approach for differentiating malignant from benign enhancing foci on breast MRI. Eur Radiol Exp. 2020 28;4(1):5.

27. Saranathan M, Rettmann DW, Hargreaves BA, Clarke SE, Vasanawala SS. DIfferential Subsampling with Cartesian Ordering (DISCO): a High Spatio-temporal Resolution Dixon Imaging Sequence for Multiphasic Contrast Enhanced Abdominal Imaging. J Magn Reson Imaging. 2012 Jun;35(6):1484-92.

28. EA Morris, CE Comstock, CH Lee. ACR BI-RADS Atlas - Breast Imaging Reporting and Data System Atlas. 2013;(Reston, VA, American College of Radiology).

29. DeLong ER, DeLong DM, Clarke-Pearson DL. Comparing the areas under two or more correlated receiver operating characteristic curves: a nonparametric approach. Biometrics. 1988 Sep;44(3):837-45.

30. Thomassin-Naggara I, Bazot M, Daraï E, Callard P, Thomassin J, Cuenod CA. Epithelial Ovarian Tumors: Value of Dynamic Contrast-enhanced MR Imaging and Correlation with Tumor Angiogenesis. Radiology. 2008 Jul 1;248(1):148-59.

31. Thomassin-Naggara I, Daraï E, Cuenod CA, Rouzier R, Callard P, Bazot M. Dynamic contrast-enhanced magnetic resonance imaging: A useful tool for characterizing ovarian epithelial tumors. J Magn Reson Imaging. 28(1):111-20.

32. Yushkevich PA, Piven J, Hazlett HC, et al. User-guided 3D active contour segmentation of anatomical structures: significantly improved efficiency and reliability. NeuroImage. 2006 Jul 1;31(3):1116-28.

33. van Griethuysen JJM, Fedorov A, Parmar C, et al. Computational Radiomics System to Decode the Radiographic Phenotype. Cancer Res. 2017 01;77(21):e104-7. 
34. Zwanenburg A, Leger S, Vallières $M$, Löck S, Initiative for the IBS. Image biomarker standardisation initiative. ArXiv161207003 Cs [Internet]. 2016 Dec 21 [cited 2018 Mar 25]; Available from: http://arxiv.org/abs/1612.07003

35. Duron L, Balvay D, Vande Perre S, et al. Gray-level discretization impacts reproducible MRI radiomics texture features. PloS One. 2019;14(3):e0213459.

36. R Core Team. R: A language and environment for statistical computing. $\mathrm{R}$ Foundation for Statistical Computing, Vienna, Austria. [Internet]. 2013. Available from: http://www.R-project.org/.

37. Tibshirani R. Regression Shrinkage and Selection via the Lasso. J R Stat Soc Ser B Methodol. 1996;58(1):267-88.

38. Lambin P, Leijenaar RTH, Deist TM, Peerlings J, de Jong EEC, van Timmeren J, et al. Radiomics: the bridge between medical imaging and personalized medicine. Nat Rev Clin Oncol. 2017 Dec;14(12):749-62.

39. Liang C, Cheng Z, Huang Y, et al. An MRI-based Radiomics Classifier for Preoperative Prediction of Ki-67 Status in Breast Cancer. Acad Radiol. 2018;25(9):1111-7. 40. Harvey SC, Di Carlo PA, Lee B, Obadina E, Sippo D, Mullen L. An Abbreviated Protocol for High-Risk Screening Breast MRI Saves Time and Resources. J Am Coll Radiol JACR. 2016 Nov;13(11S):R74-80.

41. Kuhl CK. Abbreviated breast MRI for screening women with dense breast: the EA1141 trial. Br J Radiol. 2018 Oct;91(1090):20170441.

42. Cuenod CA, Balvay D. Perfusion and vascular permeability: Basic concepts and measurement in DCE-CT and DCE-MRI. Diagn Interv Imaging. 2013 Dec 1;94(12):1187204.

43. Pradel C, Siauve N, Bruneteau G, et al. Reduced capillary perfusion and permeability in human tumour xenografts treated with the VEGF signalling inhibitor ZD4190: an in vivo assessment using dynamic MR imaging and macromolecular contrast media. Magn Reson Imaging. 2003 Oct;21(8):845-51.

44. Folkman J. Tumor Angiogenesis. In: Klein G, Weinhouse S, editors. Advances in Cancer Research [Internet]. Academic Press; 1985 [cited 2018 Jun 6]. p. 175-203. Available from: http://www.sciencedirect.com/science/article/pii/S0065230X0860946X

45. McDonald DM, Choyke PL. Imaging of angiogenesis: from microscope to clinic. Nat Med. 2003 Jun;9(6):713-25.

46. Fukumura D, Jain RK. Imaging angiogenesis and the microenvironment. APMIS Acta Pathol Microbiol Immunol Scand. 2008 Aug;116(7-8):695-715.

47. Bergers G, Benjamin LE. Tumorigenesis and the angiogenic switch. Nat Rev Cancer. 2003 Jun;3(6):401-10.

48. Reig B, Heacock L, Geras KJ, Moy L. Machine learning in breast MRI. J Magn Reson Imaging JMRI. 2019 Jul 5; 10.1002/jmri.26852

49. Agner SC, Soman S, Libfeld E, et al. Textural kinetics: a novel dynamic contrastenhanced (DCE)-MRI feature for breast lesion classification. J Digit Imaging. 2011 Jun;24(3):446-63.

50. Thibault G, Tudorica A, Afzal A, et al. DCE-MRI Texture Features for Early Prediction of Breast Cancer Therapy Response. Tomography. 2017 Mar;3(1):23-32. 51. Milenković J, Dalmış MU, Žgajnar J, Platel B. Textural analysis of early-phase spatiotemporal changes in contrast enhancement of breast lesions imaged with an ultrafast DCE-MRI protocol. Med Phys. 2017 Sep;44(9):4652-64.

52. Zhou X, Gao F, Duan S, et al. Radiomic features of Pk-DCE MRI parameters based on the extensive Tofts model in application of breast cancer. Phys Eng Sci Med. 2020 Jun;43(2):517-24.

53. Valdora F, Houssami N, Rossi F, Calabrese M, Tagliafico AS. Rapid review: 
radiomics and breast cancer. Breast Cancer Res Treat. 2018 Jun;169(2):217-29.

54. Whitney HM, Li H, Ji Y, Liu P, Giger ML. Harmonization of radiomic features of breast lesions across international DCE-MRI datasets. J Med Imaging Bellingham Wash. 2020 Jan;7(1):012707.

55. Rotili A, Trimboli RM, Penco S, et al. Double reading of diffusion-weighted magnetic resonance imaging for breast cancer detection. Breast Cancer Res Treat. 2020

Feb;180(1):111-20. 


\section{TABLE AND FIGURE LEGENDS}

Table 1 : The 57 textural features selected as reproducible regarding manual segmentation using ICC and CCC to assess variability. GLCM: Grey Level Co-occurrence Matrix GLDM: Grey Level Difference Matrix GLRLM: Grey Level Run Length Matrix GLSZM: Grey Level Size Zone Matrix

Table 2 : Comparison of diagnostic performance of the malignant probability score (results from the final model) with a threshold fixed at $28.4 \%$ and the BI-RADS classification on the conventional protocol.

Table 3 : The 15 parameters used by the algorithm in the final model "malignant probability score" and their coefficient. GLCM: Grey Level Co-occurrence Matrix GLDM: Grey Level Difference Matrix GLRLM: Grey Level Run Length Matrix

Figure 1 : Flowchart of patient inclusion and follow-up

Figure 2 : Semiquantitative enhancement parameters calculated on the native curve (a) and the curve fitted by Hill's equation (b). SI: signal intensity; sec: second EI: Enhancement Integral; Rmax: Maximum of enhancement; Rmax timing: timing of Maximum of enhancement, EA: Enhancement Amplitude, THR: Time of Half Rising

Figure 3 : Creation and selection steps of the features

Figure 4 : ROC curve of LASSO regression performed for each of the 11 phases of the HTRDCE MR sequence. ROC AUC for predicting malignancy ranged from 0.76 to 0.80 . according to the phase and were lower than that of BI-RADS analysis $(\mathrm{AUC}=0.83$ )

Figure 5 : Probability of malignancy as a function of lesion diameter for benign (green) or malignant (red) lesions

Figure 6 : a. Examples of breast MRI before and after gadolinium with the probability score of malignancy and the histological result. A. 62-year-old women with prior left breast cancer. Irregular spiculated mass on the left lower outer quadrant classified BI-RADS 4c. Malignant probability score was $5.6 \%(<28.4 \%)$. Histological analysis found a cytosteatonecrosis lesion. B. Extension assessment of lobular carcinoma; round circumscribed mass on the left lower outer quadrant classified BI-RADS 3. The malignant probability score was $33.6 \%$ (>28.4\%). Histological analysis found a lobular carcinoma. C. 37-year-old women with prior DCIS treated by mastectomy and deep inferior epigastric perforator flap (DIEP) reconstruction. An irregular mass on the right upper outer quadrant was classified BI-RADS 4a. The malignant probability score was $98 \%$ (>28.4\%). Histological analysis found a DCIS recurrence.

b. Representation of the probability score of malignancy results from 0 to $100 \%$ with the threshold of $28.4 \%$ which gave the best sensitivity-specificity rate, the result of the three examples, the $19.5 \%$ threshold which gave a $100 \%$ VPN and the $81 \%$ threshold which gave a $100 \%$ VPP. 